\title{
Existence results and two step proximal point algorithm for equilibrium problems on Hadamard manifolds
}

\author{
SUliman Al-HOMIDAN ${ }^{1}$, QAMrUl HASAN ANSARI ${ }^{1,2}$ and MONIRUl IsLAM ${ }^{2}$
}

\begin{abstract}
In this paper, we study the existence of solutions of equilibrium problems in the setting of Hadamard manifolds under the pseudomonotonicity and geodesic upper sign continuity of the equilibrium bifunction and under different kinds of coercivity conditions. We also study the existence of solutions of the equilibrium problems under properly quasimonotonicity of the equilibrium bifunction. We propose a two-step proximal point algorithm for solving equilibrium problems in the setting of Hadamard manifolds. The convergence of the proposed algorithm is studied under the strong pseudomonotonicity and Lipschitz-type condition. The results of this paper either extend or generalize several known results in the literature.
\end{abstract}

\section{INTRODUCTION}

It is well-known that the equilibrium problem is a unified model of several mathematical problems, namely, variational inequality problems, hemivariational inequality problems, complementarity problems, fixed point problems, saddle point problems, optimization problems, Nash equilibrium problems, etc, see, for example $[5,10,14]$ and the references therein. Kim et al. [15] proposed a proximal point-type algorithm for pseudomonotone equilibrium problems in the setting of finite dimensional Euclidean space $\mathbb{R}^{n}$, and studied its convergence without Lipschitizian assumption. Recently, two-step proximal algorithm for solving equilibrium problems has been introduced and studied in $[12,19]$ under the pseudomonotonicity and Lipschitz-type condition of the underlying equilibrium bifunction. Hieu [11] considered two-step algorithm with underlying constant is not fixed but it is a sequence of positive real numbers, and studied the convergence of the sequence generated by the algorithm under strongly pseudomonotonicity and Lipschitz-type condition.

During the last decade, several known theories and methods from optimization and nonlinear analysis have been extended from linear structures to Riemannian / Hadamard manifolds because some constrained optimization problems can be seen as unconstrained ones from Riemannian geometry point of view. Colao et al. [6] extended well-known KKM lemma [16] from Euclidean space to Hadamard manifolds. By using this result, they proved the existence of solutions of the equilibrium problems defined on the Hadamard manifold. As consequences, they derived the existence results for solutions of mixed variational inequality problems, fixed point problems for set-valued mappings and Nash equilibrium problem for noncooperative games. They also studied the convergence of Picard iteration for firmly nonexpansive mappings along with the definition of resolvents for equilibrium bifunction which is used to propose an algorithm for solving equilibrium problems in this setting. Ferreira and Oliveira [9] extended proximal point method for solving optimization problems from linear structure to Riemannian manifolds (see,

Received: 12.06.2020. In revised form: 25.10.2020. Accepted: 14.06.2020

2010 Mathematics Subject Classification. 49J40, 65K15, 65K10, 47J25, 47J20.

Key words and phrases. Equilibrium problems, Existence results, Coercivity conditions, Two-step proximal point algorithm, Lipschitz-type condition, Hadamard manifolds.

Corresponding author: Qamrul Hasan Ansari; qhansari@gmail.com 
also [4]). Recently, Cruz Neto et al. [7] proposed extragradient algorithm for solving equilibrium problems on Hadamard manifolds where the equilibrium bifunction is not necessarily pseudomonotone.

In this paper, we consider the equilibrium problems in the setting of Hadamard manifolds and study the existence of their solutions under pseudomonotonicity and geodesic upper sign continuity of the equilibrium bifunction. We extend the two-step proximal point algorithm [11] from Hilbert space to Hadamard manifolds setting.

The present paper is organized as follows: In the next section, we collect some basic definitions, notions and results from manifolds. The formulations of equilibrium problem and dual equilibrium problem are presented, and the equivalence of these problems is also studied under pseudomonotonicity and geodesic upper sign continuity of the equilibrium bifunction. Section 3 deals with the existence of solutions of equilibrium problems and dual equilibrium problems without compactness assumption on the underlying set but under different kinds of coercivity conditions. In the last section, we present a twostep proximal point algorithm for solving equilibrium problems on Hadamard manifolds. We study the convergence of the sequences generated by the proposed algorithm to a solution of the equilibrium problem under strong pseudomonotonicity and Lipschitz-type condition. The results of this paper either improve or extend several known results in the literature.

\section{PRELIMINARIES}

2.1. Elements from manifolds. We recall some known definitions, notations, concepts and results from manifolds which can be found in any standard book on manifolds, see, for example $[8,23,24,26]$.

Let $M$ be a finite dimensional differentiable manifold and $T_{x} M$ be the tangent space at the point $x \in M$ to $M$. Such tangent space forms a real vector space of the same dimension as $M$. The collection of all the tangent spaces to $M$ is called a tangent bundle of $M$ and it is denoted by $T M$.

A $C^{\infty}$ mapping $V: M \rightarrow T M$ which assigns a tangent vector $V(x)$ at $x$ for each $x \in M$, is called a vector field on $M$.

A scalar product $\langle\cdot, \cdot\rangle_{x}$ on $T_{x} M$ is called Riemannian metric on $T_{x} M$ and the associated norm is denoted by $\|\cdot\|_{x}$. If no ambiguity occurs, then we omit the subscript $x$,

Let $g$ be a $C^{\infty}$ tensor field of type $(0,2)$ on $M$. If for each $x \in M$, the tensor $g(x)$ is a Riemannian metric on $T_{x} M$, then the tensor field $g$ is called Riemannian metric on $M$ and the pair $(M, g)$ is called the Riemannian manifold. By using the Riemannian metric, the length of a piecewise smooth curve $\gamma:[0,1] \rightarrow M$ joining $x$ to $y$ such that $\gamma(0)=x$ and $\gamma(1)=y$, is defined as $L(\gamma):=\int_{0}^{1}\|\dot{\gamma}(t)\|_{\gamma(t)} d t$, where $\dot{\gamma}(t)$ denotes the tangent vector at $\gamma(t)$. The minimum length of the function $L(\gamma)$ over the set of all piecewise smooth curves joining $x$ to $y$ is called the Riemannian distance and it is denoted by $d(x, y)$.

Let $\nabla$ be the Levi-Civita (or Riemannian) connection on $M$. A curve $\gamma:[0,1] \rightarrow M$ joining $x$ to $y$ such that $\gamma(0)=x, \gamma(1)=y$ and $\nabla_{\dot{\gamma}(t)} \dot{\gamma}(t)=0$ on $[0,1]$, is called a geodesic. To emphasis the initial point $\gamma(0)=x$ and terminal point $\gamma(1)=y$ of the geodesic $\gamma$, some time we write $\gamma_{x y}$ instead of $\gamma$.

A geodesic $\gamma$ joining $x$ to $y$ in $M$ is said to be minimal if its length equals its Riemannian distance between $x$ and $y$. If for any $x \in M$, all geodesic emanating from $x$ are defined for all $t \in \mathbb{R}$, then the Riemannian manifold $M$ is said to be complete.

A simply connected complete Riemannian manifold $M$ with nonpositive sectional curvature is called a Hadamard manifold. 
The exponential map $\exp _{x}: T_{x} M \rightarrow M$ at $x$ is defined by $\exp _{x} v=\gamma_{v}(1, x)$ for each $v \in T_{x} M$, where $\gamma(\cdot)=\gamma_{v}(\cdot, x)$ is the geodesic starting from $x$ with the velocity $v$, that is, $\gamma(0)=x$ and $\dot{\gamma}(0)=v$. Moreover, $\exp _{x} t v=\gamma_{v}(t, x)$ for each real number $t$.

We denote by $P_{\gamma(b), \gamma(a)}$ the parallel transport from $T_{\gamma(a)} M$ to $T_{\gamma(b)} M$ along the geodesic $\gamma$ with respect to $\nabla$ and defined by

$$
P_{\gamma(b), \gamma(a)}(v)=V(\gamma(b)), \quad \text { for all } a, b \in \mathbb{R} \text { and } v \in T_{\gamma(a)} M,
$$

where $V$ is the unique vector field such that $\nabla_{\dot{\gamma}(t)} V=0$ for all $t$ and $V(\gamma(a))=v$.

Proposition 2.1. [8] Let $M$ be a Hadamard manifold. For any given $x \in M$, $\exp _{x}: T_{x} M \rightarrow M$ is a diffeomorphism. Furthermore, for any two points $x, y \in M$, there exists a unique minimal geodesic $\gamma$ joining $x$ to $y$ such that $\gamma(t)=\exp _{x}\left(t \exp _{x}^{-1} y\right)$ for all $t \in[0,1]$. In particular, the exponential map and its inverse are continuous on a Hadamard manifold.

A geodesic triangle $\triangle\left(x_{1}, x_{2}, x_{3}\right)$ of a Riemannian manifold $M$ is a set consisting of three points $x_{1}, x_{2}$ and $x_{3}$, and three minimal geodesics $\gamma_{i}$ joining $x_{i}$ to $x_{i+1}$ with $i=1,2,3$ $(\bmod 3)$.

Proposition 2.2. [23] Let $\triangle\left(x_{1}, x_{2}, x_{3}\right)$ be a geodesic triangle in a Hadamard manifold $M$. For each $i=1,2,3(\bmod 3)$, let $\gamma_{i}:\left[0, l_{i}\right] \rightarrow M$ be the geodesic segment joining $x_{i}$ to $x_{i+1}$, and set $l_{i}=L\left(\gamma_{i}\right)$ and $\theta_{i}=\angle\left(\dot{\gamma}_{i}(0),-\dot{\gamma}_{i-1}\left(l_{i-1}\right)\right)$ the angle between the vector $\dot{\gamma}_{i}(0)$ and $-\dot{\gamma}_{i-1}\left(l_{i-1}\right)$. Then

$$
\theta_{1}+\theta_{2}+\theta_{3} \leq \pi
$$

and

$$
l_{i}^{2}+l_{i+1}^{2}-2 l_{i} l_{i+1} \cos \theta_{i+1} \leq l_{i-1}^{2} .
$$

Since $\left\langle\exp _{x_{i+1}}^{-1} x_{i}, \exp _{x_{i+1}}^{-1} x_{i+2}\right\rangle=d\left(x_{i}, x_{i+1}\right) d\left(x_{i+1}, x_{i+2}\right) \cos \theta_{i+1}$ for all $i=1,2,3(\bmod 3)$ (see [23]), the inequality (2.2) can be re-written in terms of the Riemannian distance and exponential map as

$$
d^{2}\left(x_{i}, x_{i+1}\right)+d^{2}\left(x_{i+1}, x_{i+2}\right)-2\left\langle\exp _{x_{i+1}}^{-1} x_{i}, \exp _{x_{i+1}}^{-1} x_{i+2}\right\rangle \leq d^{2}\left(x_{i-1}, x_{i}\right) .
$$

Lemma 2.1. [22] Let $\triangle\left(x_{1}, x_{2}, x_{3}\right)$ be a geodesic triangle in a Hadamard manifold $M$. Then there exists $x_{1}^{\prime}, x_{2}^{\prime}, x_{3}^{\prime} \in \mathbb{R}^{2}$ such that

$$
d\left(x_{1}, x_{2}\right)=\left\|x_{1}^{\prime}-x_{2}^{\prime}\right\|, \quad d\left(x_{2}, x_{3}\right)=\left\|x_{2}^{\prime}-x_{3}^{\prime}\right\| \quad \text { and } \quad d\left(x_{3}, x_{1}\right)=\left\|x_{3}^{\prime}-x_{1}^{\prime}\right\| .
$$

The triangle $\triangle\left(x_{1}^{\prime}, x_{2}^{\prime}, x_{3}^{\prime}\right)$ is called the comparison triangle of the geodesic triangle $\triangle\left(x_{1}, x_{2}, x_{3}\right)$, which is unique up to isometry of $M$. The points $x_{1}^{\prime}, x_{2}^{\prime}, x_{3}^{\prime}$ are called comparison points of the points $x_{1}, x_{2}, x_{3}$, respectively.

Lemma 2.2. [17] Let $\triangle\left(x_{1}, x_{2}, x_{3}\right)$ be a geodesic triangle in a Hadamard manifold $M$ and $\triangle\left(x_{1}^{\prime}, x_{2}^{\prime}, x_{3}^{\prime}\right)$ be its comparison triangle.

(a) Let $\theta_{1}, \theta_{2}, \theta_{3}$ (respectively, $\left.\theta_{1}^{\prime}, \theta_{2}^{\prime}, \theta_{3}^{\prime}\right)$ be the angles of $\triangle\left(x_{1}, x_{2}, x_{3}\right)$ (respectively, $\triangle\left(x_{1}^{\prime}, x_{2}^{\prime}, x_{3}^{\prime}\right)$ ) at vertices $x_{1}, x_{2}, x_{3}$ (respectively, $x_{1}^{\prime}, x_{2}^{\prime}, x_{3}^{\prime}$ ). Then

$$
\theta_{1}^{\prime} \geq \theta_{1}, \quad \theta_{2}^{\prime} \geq \theta_{2} \quad \text { and } \quad \theta_{3}^{\prime} \geq \theta_{3} .
$$

(b) Let $\bar{x}$ be the point on the geodesic joining $x_{1}$ to $x_{2}$ and $\bar{x}^{\prime}$ its comparison point on the line segment $\left[x_{1}^{\prime}, x_{2}^{\prime}\right]$. Suppose that $d\left(\bar{x}, x_{1}\right)=\left\|\bar{x}^{\prime}-x_{1}^{\prime}\right\|$ and $d\left(\bar{x}, x_{2}\right)=\left\|\bar{x}^{\prime}-x_{2}^{\prime}\right\|$. Then

$$
d\left(\bar{x}, x_{3}\right) \leq\left\|\bar{x}^{\prime}-x_{3}^{\prime}\right\| \text {. }
$$


A subset $K$ of a Hadamard manifold $M$ is said to be geodesic convex if for any pair of distinct points $x, y \in K$, the geodesic $\gamma$ joining $x$ to $y$ belongs to $K$, that is, if for any $\gamma:[0,1] \rightarrow M$ such that $\gamma(0)=x$ and $\gamma(1)=y$, then $\gamma(t)=\exp _{x}\left(t \exp _{x}^{-1} y\right) \in K$ for all $t \in[0,1]$.

Let $C$ be a subset of a Hadamard manifold $M$. The smallest geodesic convex subset of $M$ that contains $C$ is called the convex hull of $C$, and it is denoted by $\operatorname{co}(C)$. As pointed out in [6] that $\operatorname{co}(C)=\bigcup_{n=1}^{\infty} C_{n}$, where $C_{0}=C$ and $C_{n}=\left\{z \in \gamma_{x, y}: x, y \in C_{n-1}\right\}$, where $\gamma_{x, y}$ denotes the geodesic joining $x$ to $y$.

Zhou et al. [27] gave the following geometrical definition of geodesic convex combination of finite number of points in terms of exp.

The following two theorems provide the relationship among geodesic convex combination, geodesic convex set and geodesic convex hull.

Theorem 2.1. [27] Let $M$ be a Hadamard manifold. A set $K \subset M$ is geodesic convex if and only if it contains all the geodesic convex combinations of its elements.

Theorem 2.2. [27] Let $K \subset M$ be any set in a Hadamard manifold $M$. Then $\operatorname{co}(K)$ consists of all the geodesic combinations of elements of $K$.

Theorem 2.3. [27] For any two subsets $K_{1}, K_{2}$ of a Hadamard manifold $M$ such that $K_{1} \subseteq K_{2}$, then $\operatorname{co}\left(K_{1}\right) \subseteq \operatorname{co}\left(K_{2}\right)$.

Let $K$ be a geodesic convex subset of a Hadamard manifold $M$. A real-valued function $f: K \rightarrow \mathbb{R}$ is said to be geodesic convex if

$$
f\left(\exp _{x}\left(t \exp _{x}^{-1} y\right)\right) \leq(1-t) f(x)+t f(y), \quad \text { for all } x, y \in K \text { and all } t \in[0,1] .
$$

Colao et al. [6] extended the well-known KKM lemma [16] from finite dimensional space to Hadamard manifold settings and used it to prove the existence of solutions of equilibrium problems.

Let $K$ be a geodesic convex subset of a Hadamard manifold $M$. A set-valued mapping $T: K \rightrightarrows K$ is said to be a KKM mapping if for any finite subset $\left\{x_{1}, x_{2}, \ldots, x_{m}\right\}$ of $K$, $\operatorname{co}\left(\left\{x_{1}, x_{2}, \ldots, x_{m}\right\}\right) \subset \bigcup_{i=1}^{m} T\left(x_{i}\right)$.

Lemma 2.3. [6] Let $K$ be a nonempty geodesic convex subset of a Hadamard manifold $M$ and $T: K \rightrightarrows K$ be a KKM mapping such that for each $x \in K, T(x)$ is closed and for at least one $x_{0} \in K, T\left(x_{0}\right)$ is compact. Then $\bigcap_{x \in K} T(x) \neq \emptyset$.

2.2. Formulation of the problems. Let $K$ be a nonempty geodesic convex subset of a Hadamard manifold $M$ and $f: K \times K \rightarrow \mathbb{R}$ be a real-valued bifunction such that $f(x, x) \geq$ 0 for all $x \in K$. The equilibrium problem (in short, EP) is to find $\bar{x} \in K$ such that

$$
f(\bar{x}, y) \geq 0, \quad \text { for all } y \in K .
$$

It includes several problems, namely, optimization problems, variational inequality problems [21], hemivariational inequality problems [25], nonsmooth variational inequality problems [2], fixed point problems, Nash equilibrium problem, as special cases, see, for example [5]. Colao et al. [6] first considered the equilibrium problem in the setting of Hadamard manifolds and studied the existence of its solutions. They also suggested Picard iterative method for finding the solution of EP.

We also consider the following problem which is closely related to the equilibrium problem and known as dual equilibrium problem or Minty equilibrium problem.

Find $\bar{x} \in K$ such that $f(y, \bar{x}) \leq 0, \quad$ for all $y \in K$.

Let $V: K \rightarrow T M$ be a vector filed, that is, $A(x) \in T_{x} M$ for all $x \in K$, and $J: M \rightarrow \mathbb{R}$ be a locally Lipschitz function, that is, for each $x$ in the domain of $J$, there exist $\varepsilon_{x}>0$ 
and $L_{x}>0$ such that

$$
|J(z)-J(y)| \leq L_{x} d(z, y), \quad \text { for all } z, y \in \mathbb{B}\left(x, \varepsilon_{x}\right),
$$

where $\mathbb{B}\left(x, \varepsilon_{x}\right)$ denotes an open ball centered at $x \in M$ and radius $\varepsilon_{x}$, that is, $\mathbb{B}\left(x, \varepsilon_{x}\right):=$ $\left\{y \in M: d(x, y)<\varepsilon_{x}\right\}$. Tang et al. [25] considered the following hemivariational inequality problem: Find $\bar{x} \in K$ such that

$$
\left\langle V(\bar{x}), \exp _{\bar{x}}^{-1} y\right\rangle+J^{\circ}\left(\bar{x} ; \exp _{\bar{x}}^{-1} y\right) \geq 0, \quad \text { for all } y \in K,
$$

where $J^{\circ}(x ; w)$ denotes the Clarke directional derivative at the point $x \in K$ and in the direction $w \in T_{x} M$, [13]. The hemivariational inequality problem contains several kinds of variational inequality problems considered and studied in $[2,17,18,21]$ and the references therein.

If $J^{\circ}(x ; w)=0$ for all $x$ and $w$, then the hemivariational inequality problem (2.8) reduces to the following variational inequality problem, considered and studied by Németh [21]: Find $\bar{x} \in K$ such that

$$
\left\langle V(\bar{x}), \exp _{\bar{x}}^{-1} y\right\rangle \geq 0, \quad \text { for all } y \in K .
$$

Definition 2.1. A bifunction $f: K \times K \rightarrow \mathbb{R}$ is said to be

(a) monotone if

$$
f(x, y)+f(y, x) \leq 0, \quad \text { for all } x, y \in K ;
$$

(b) pseudomonotone if

$$
f(x, y) \geq 0 \Longrightarrow f(y, x) \leq 0, \quad \text { for all } x, y \in K ;
$$

(c) strongly pseudomonotone if there exists a constant $\lambda>0$ such that

$$
f(x, y) \geq 0 \Longrightarrow f(y, x) \leq-\lambda d^{2}(x, y), \quad \text { for all } x, y \in K .
$$

Clearly, monotonicity implies pseudomonotonicity and strongly pseudomonotonicity implies pseudomonotonicity.

Definition 2.2. Let $K$ be a nonempty geodesic convex subset of a Hadamard manifold $M$. A bifunction $f: K \times K \rightarrow \mathbb{R}$ is said to be geodesic upper sign continuous if for every $x, y \in K$, we have

$$
f\left(w_{t}, x\right) \leq 0 \Longrightarrow f(x, y) \geq 0,
$$

where $w_{t}=\exp _{x} t \exp _{x}^{-1} y$ for all $t \in(0,1)$.

Definition 2.3. [1] Let $K$ be a nonempty geodesic convex subset of a Hadamard manifold $M$. A function $g: K \rightarrow \mathbb{R}$ is said to be geodesic hemicontinuous if for any geodesic $\gamma:[0,1] \rightarrow M$, the function $t \mapsto g(\gamma(t))$ defined on $[0,1]$ is continuous, that is, $g(\gamma(t)) \rightarrow$ $g(\gamma(0))$ as $t \rightarrow 0$.

Definition 2.4. [21] Let $K$ be a nonempty geodesic convex subset of a Hadamard manifold $M$. A vector field $V$ on $K$ is said to be geodesic hemicontinous if for every geodesic $\gamma:[0,1] \rightarrow K$ and $w \in T_{\gamma(0)} M$, the function $t \mapsto\left\langle P_{\gamma(0), \gamma(t)} V(\gamma(t)), w\right\rangle$ is continous.

Remark 2.1. Let $V: K \rightarrow T M$ be a vector field such that $g(\gamma(t))=\left\langle P_{\gamma(0), \gamma(t)} V(\gamma(t)), w\right\rangle$ for any geodesic $\gamma:[0,1] \rightarrow M$ and $w \in T_{\gamma(0)} M$. Then geodesic hemicontinuity of $g$ implies the geodesic hemicontinuity of $V$.

Remark 2.2. If the bifunction $f: K \times K \rightarrow \mathbb{R}$ is geodesic hemicontinuous in the first argument and geodesic convex in the second argument, then it is geodesic upper sign continuous. 
Indeed, assume that for every $x, y \in K$, we have

$$
f\left(w_{t}, x\right) \leq 0, \quad \text { for all } t \in(0,1) .
$$

Since $f(x, x) \geq 0$ for all $x \in K$ and $f$ is geodesic convex in the second argument, we have

$$
0 \leq f\left(w_{t}, w_{t}\right) \leq(1-t) f\left(w_{t}, x\right)+t f\left(w_{t}, y\right) \leq t f\left(w_{t}, y\right), \quad \text { for all } t \in(0,1) .
$$

As $t>0$, we obtain $f\left(w_{t}, y\right) \geq 0$. Since the bifunction $f$ is geodesic hemicontinuous in the first argument, we have $f(x, y) \geq 0$.

The following lemma provides the equivalence between EP (2.6) and DEP (2.7).

Lemma 2.4. Let $K$ be a nonempty geodesic convex subset of a Hadamard manifold $M$ and $f$ : $K \times K \rightarrow \mathbb{R}$ be a pseudomonotone and geodesic upper sign continuous bifunction. Then $\bar{x} \in K$ is a solution of EP (2.6) if and only if it is a solution of DEP (2.7).

Proof. Clearly from pseudomonotonicity of $f$, every solution of EP (2.6) is a solution of $\operatorname{DEP}$ (2.7). For the converse, assume that $\bar{x} \in K$ is a solution of $\operatorname{DEP}(2.7)$. Then $f(y, \bar{x}) \leq 0$ for all $y \in K$. Since $K$ is geodesic convex, we have $w_{t}=\exp _{\bar{x}} t \exp _{\bar{x}}^{-1} y \in K$ for all $t \in(0,1)$. Therefore, $f\left(w_{t}, \bar{x}\right) \leq 0$. By geodesic upper sign continuity of $f$, we get $f(\bar{x}, y) \geq$ 0 .

\section{EXISTENCE RESULTS FOR SOLUTIONS}

We establish some existence results for solutions of EP (2.6) and DEP (2.7) under different conditions on the underlying set $K$ and the bifunction $f$.

Theorem 3.4. Let $K$ be a nonempty closed and geodesic convex subset of a Hadamard manifold $M$ and $f: K \times K \rightarrow \mathbb{R}$ be a pseudomonotone and geodesic upper sign continuous bifunction such that the following conditions hold:

(i) For all $x \in K, f(x, x) \geq 0$;

(ii) For all $x \in K$, the set $\{y \in K: f(x, y)<0\}$ is geodesic convex;

(iii) For all $y \in K$, the function $x \mapsto f(y, x)$ is lower semicontinuous;

(iv) There exist a compact subset $C$ of $M$ and $\tilde{y} \in K \cap C$ such that

$$
f(x, \tilde{y})<0, \quad \text { for all } x \in K \backslash C .
$$

Then there exists a solution $\bar{x} \in K$ of EP (2.6).

Proof. For all $y \in K$, define $P, Q: K \rightrightarrows K$ by

$$
P(y)=\{x \in K: f(x, y) \geq 0\}
$$

and

$$
Q(y)=\{x \in K: f(y, x) \leq 0\}
$$

As in [6], $P$ is a KKM mapping. We include it for the sake of completeness.

Suppose contrary that there exist a finite subset $\left\{y_{1}, y_{2}, \ldots, y_{m}\right\}$ of $K$ and a point $\hat{x} \in$ $\operatorname{co}\left(\left\{y_{1}, y_{2}, \ldots, y_{m}\right\}\right)$ such that $\hat{x} \notin \bigcup_{i=1}^{m} P\left(y_{i}\right)$, that is,

$$
f\left(\hat{x}, y_{i}\right)<0, \quad \text { for all } i=1,2, \ldots, m .
$$

Then $y_{i} \in\{y \in K: f(\hat{x}, y)<0\}$ for each $i=1,2, \ldots, m$. By (ii), the set $\{y \in K: f(\hat{x}, y)<$ $0\}$ is geodesic convex, and therefore by Theorem 2.3, we have $\hat{x} \in \operatorname{co}\left(\left\{y_{1}, y_{2}, \ldots, y_{m}\right\}\right) \subseteq$ $\{y \in K: f(\hat{x}, y)<0\}$, that is, $f(\hat{x}, \hat{x})<0$ which contradicts (i). Hence, $P$ is a KKM mapping.

For each $y \in K, Q(y)$ is closed. Indeed, let $\left\{x_{n}\right\} \subseteq Q(y)$ be a sequence such that $x_{n} \rightarrow x \in K$ as $n \rightarrow \infty$. We show that $x \in Q(y)$. Since $x_{n} \in Q(y)$ for each $n \in \mathbb{N}$, we have 
$f\left(y, x_{n}\right) \leq 0$. By condition (iii), we have $f(y, x) \leq \liminf _{n \rightarrow \infty} f\left(y, x_{n}\right) \leq 0$. Thus, $f(y, x) \leq 0$, and hence, $x \in Q(y)$. Therefore, $Q(y)$ is closed for each $y \in K$.

Let $\tilde{y} \in K$ and $C$ be same as in condition (iv). We want to show that $P(\tilde{y})$ is compact. If $P(\tilde{y}) \nsubseteq C$, then there exists $x \in P(\tilde{y})$ such that $x \in K \backslash C$, and hence, $f(x, \tilde{y}) \geq 0$, which contradicts (3.11). Therefore, $P(\tilde{y}) \subseteq C$. Thus the closure of $P(\tilde{y}), \operatorname{cl}(P(\tilde{y}))$ is a closed subset of the compact set $C$, hence compact. Therefore, by Lemma 2.3, we have

$$
\bigcap_{y \in K} \operatorname{cl} P(y) \neq \emptyset \text {. }
$$

By pseudomonotonicity of $f$, we have $P(y) \subseteq Q(y)$, therefore, $\operatorname{cl}(P(y)) \subseteq \operatorname{cl}(Q(y))=Q(y)$. Thus,

$$
\emptyset \neq \bigcap_{y \in K} \operatorname{cl}(P(y)) \subseteq \bigcap_{y \in K} Q(y) .
$$

Hence, there exists $\bar{x} \in K$ such that

$$
f(y, \bar{x}) \leq 0, \quad \text { for all } y \in K .
$$

By Lemma 2.4, $\bar{x} \in K$ is a solution of EP (2.6).

Remark 3.3. Colao et al. [6] proved the existence of solution of EP (2.6) by considering (i), (ii), (iv) and the following condition:

(iii') For all $y \in K, x \mapsto f(x, y)$ is upper semicontinuous.

If we consider $f(x, y)=\left\langle V(x), \exp _{x}^{-1} y\right\rangle$, then the condition (iii') is very strong. However, in this case, condition (iii) of Theorem 3.4 automatically holds, that is, $x \mapsto\left\langle V(y), \exp _{y}^{-1} x\right\rangle$ is lower semicontinuous.

Remark 3.4. The coercivity condition (iv) in Theorem 3.4 can be replaced by the following condition.

$\left(\mathrm{iv}^{\prime}\right)$ There exist a compact subset $D$ of $M$ and $\tilde{y} \in K \cap D$ such that

$$
f(\tilde{y}, x)>0, \quad \text { for all } x \in K \backslash D .
$$

Proof. Let $P$ and $Q$ be the same as defined in the proof of Theorem 3.4. Since $P$ is a KKM mapping and $P(x) \subseteq Q(x)$ by pseudomonotonicity, we have that $Q$ is also a KKM mapping. We have already proved in the proof of Theorem 3.4 that for each $y \in K, Q(y)$ is closed.

Now we shall show that $Q(\tilde{y}) \subseteq K \cap D$. If $Q(\tilde{y}) \nsubseteq D$, then there exists $x \in Q(\tilde{y})$ such that $x \in K \backslash D$, and hence, $f(\tilde{y}, x) \leq 0$ which contradicts (3.15). Thus $Q(\tilde{y}) \subseteq D$, and therefore, $Q(\tilde{y}) \subseteq K \cap D$. Since $K$ is closed and $D$ is compact, $K \cap D$ is compact. Therefore, $Q(\tilde{y})$ is a closed subset of a compact set $K \cap D$, and hence $Q(\tilde{y})$ is compact. By Lemma 2.3, we have $\bigcap_{y \in K} Q(y) \neq \emptyset$. Therefore, there exists $\bar{x} \in K$ such that $\bar{x} \in Q(y)$ for all $y \in K$, that is,

$$
f(y, \bar{x}) \leq 0, \quad \text { for all } y \in K .
$$

By Lemma 2.4, $\bar{x} \in K$ is a solution of EP (2.6).

Remark 3.5. Let $V: K \rightarrow T M$ be monotone, that is,

$$
\left\langle V(x), \exp _{x}^{-1} y\right\rangle \leq\left\langle V(y),-\exp _{y}^{-1} x\right\rangle, \quad \text { for all } x, y \in K,
$$

and a geodesic hemicontinuous vector field. Let $J: M \rightarrow \mathbb{R}$ be a locally Lipschitz function. For all $x, y \in K$, let

$$
f(x, y)=\left\langle V(x), \exp _{x}^{-1} y\right\rangle+J^{\circ}\left(x ; \exp _{x}^{-1} y\right)
$$


and

$$
f(y, x)=\left\langle V(y), \exp _{y}^{-1} x\right\rangle-J^{\circ}\left(x ; \exp _{x}^{-1} y\right) .
$$

We show that $f$ is pseudomonotone and geodesic upper sign continuous. For this, we first show that $f$ is monotone. Since $V$ is a monotone vector field, we have

$$
\begin{aligned}
f(x, y) & =\left\langle V(x), \exp _{x}^{-1} y\right\rangle+J^{\circ}\left(x ; \exp _{x}^{-1} y\right) \\
& \leq-\left\langle V(y),-\exp _{y}^{-1} x\right\rangle+J^{\circ}\left(x ; \exp _{x}^{-1} y\right) \\
& =-\left\{\left\langle V(y), \exp _{y}^{-1} x\right\rangle-J^{\circ}\left(x ; \exp _{x}^{-1} y\right)\right\}=-f(y, x),
\end{aligned}
$$

which shows that $f$ is monotone, and hence pseudomonotone.

Now we show that $f$ is geodesic upper sign continuous, that is, $f$ satisfies inequality (2.9). For any $x, y \in K$ and any geodesic $\gamma:[0,1] \rightarrow M$ joining $x$ to $y$, that is, $\gamma(t)=$ $\exp _{x} t \exp _{x}^{-1} y$ for $t \in(0,1)$, assume that $f(\gamma(t), x) \leq 0$ for all $t \in(0,1)$. Then

$$
\begin{aligned}
0 & \geq f\left(\exp _{x} t \exp _{x}^{-1} y, x\right) \\
& =\left\langle V\left(\exp _{x} t \exp _{x}^{-1} y\right), \exp _{\exp _{x} t \exp _{x}^{-1} y}^{-1} x\right\rangle-J^{\circ}\left(x ; \exp _{x}^{-1}\left(\exp _{x} t \exp _{x}^{-1} y\right)\right) \\
& \left.=\left\langle V\left(\exp _{x} t \exp _{x}^{-1} y\right), \exp _{\exp _{x} t \exp _{x}^{-1} y}^{-1} x\right\rangle-J^{\circ}\left(x ; t \exp _{x}^{-1} y\right)\right) .
\end{aligned}
$$

Since the parallel transport along a curve is an isometry and the tangent vector of the geodesic is parallel along the geodesic and $J^{\circ}$ is positively homogeneous in the second argument, we obtain

$$
0 \geq t\left\langle P_{\gamma(0), \gamma(t)} V\left(\exp _{x} t \exp _{x}^{-1} y\right),-\exp _{x}^{-1} y\right\rangle-t J^{\circ}\left(x ; \exp _{x}^{-1} y\right) .
$$

Since $t>0$, and $V$ is geodesic hemicontinuous vector field, we have

$$
\left\langle V(x), \exp _{x}^{-1} y\right\rangle+J^{\circ}\left(x ; \exp _{x}^{-1} y\right) \geq 0,
$$

that is, $f(x, y) \geq 0$. Hence $f$ is geodesic upper sign continuous.

Therefore, by using Theorem 3.4, we can obtain an existence result for a solution of hemivariational inequality problem (2.8). Also, Theorem 3.4 generalizes Theorem 3.3 in [25].

Definition 3.5. Let $K$ be a nonempty subset of a Hadamard manifold $M$. A bifunction $f: K \times K \rightarrow \mathbb{R}$ is said to be properly quasimonotone if for any $x_{1}, x_{2}, \ldots, x_{m} \in K$ and any $y \in \operatorname{co}\left(\left\{x_{1}, x_{2}, \ldots, x_{m}\right\}\right)$, there exists $i \in\{1,2, \ldots, m\}$ such that $f\left(x_{i}, y\right) \leq 0$.

Now we prove the existence of solution of DEP (2.7) under properly quasimonotonicity.

Theorem 3.5. Let $K$ be a nonempty closed and geodesic convex subset of a Hadamard manifold $M$ and $f: K \times K \rightarrow \mathbb{R}$ be properly quasimonotone and for each $y \in K$, the function $x \mapsto f(y, x)$ is lower semicontinuous. Assume that there exist a compact subset $D$ of $M$ and $\tilde{y} \in K \cap D$ such that

$$
f(\tilde{y}, x)>0, \quad \text { for all } x \in K \backslash D .
$$

Then DEP (2.7) has a solution.

Proof. For each $y \in K$, define the set-valued mapping $P: K \rightrightarrows K$ by

$$
P(y)=\{x \in K: f(y, x) \leq 0\} .
$$

For any $y_{1}, y_{2}, \ldots, y_{m} \in K$, let $\bar{y} \in \operatorname{co}\left(\left\{y_{1}, y_{2}, \ldots, y_{m}\right\}\right)$. Since $f$ is properly quasimonotone, we have $\bar{y} \in \bigcup_{i=1}^{m} P\left(y_{i}\right)$, that is, $P$ is a KKM mapping. By lower semicontinuity of the map $x \mapsto f(y, x), P(y)$ is a closed subset of $K$. Now we shall show that $P(\tilde{y}) \subseteq K \cap D$. If $P(\tilde{y}) \nsubseteq D$, then there exists $x \in P(\tilde{y})$ such that $x \in K \backslash D$, and hence, $f(\tilde{y}, x) \leq 0$ which contradicts (3.16). Thus $P(\tilde{y}) \subseteq D$, and therefore, $P(\tilde{y}) \subseteq K \cap D$. Since $K$ is closed and $D$ 
is compact, $K \cap D$ is compact. Therefore, $P(\tilde{y})$ is a closed subset of a compact set $K \cap D$, and hence $P(\tilde{y})$ is compact. By Lemma 2.3, we have $\bigcap_{y \in K} P(y) \neq \emptyset$. Therefore, there exists $\bar{x} \in K$ such that $\bar{x} \in P(y)$ for all $y \in K$, that is, $f(y, \bar{x}) \leq 0$ for all $y \in K$. Hence, $\bar{x} \in K$ is a solution of DEP (2.7).

Remark 3.6. Under the assumptions of Lemma 2.4 and Theorem 3.5, we can obtain the existence result for a solution of EP (2.6) without assuming condition (ii) of Theorem 3.4, that is, without assuming that the set $\{y \in K: f(x, y)<0\}$ is geodesic convex for all $x \in K$.

\section{TWO STEP PROXIMAL POINT ALGORITHM AND CONVERGENCE RESULTS}

Let $K$ be a nonempty closed geodesic convex subset of a Hadamard manifold $M, h$ : $K \rightarrow \mathbb{R}$ be a proper, geodesic convex and lower semicontinuous function and $\lambda>0$. The proximal mapping of the function $h: K \rightarrow \mathbb{R}$ is defined as follows:

$$
\operatorname{prox}_{\lambda h}(x)=\operatorname{argmin}\left\{\lambda h(y)+\frac{1}{2} d^{2}(x, y): y \in K\right\}, \quad \text { for all } x \in M \text {. }
$$

To propose the two step proximal point algorithm for finding the solutions of EP, we establish the following result which can be seen as an extension of Proposition 12.26 in [3] from Hilbert space to Hadamard manifold settings.

Lemma 4.5. Let $h: M \rightarrow(-\infty,+\infty]$ be proper, lower semicontinuous and geodesic convex function on $M$ and $x, p \in M$. Then for $\lambda>0$,

$$
p=\operatorname{prox}_{\lambda h}(x) \Leftrightarrow\left\langle\exp _{p}^{-1} y, \exp _{p}^{-1} x\right\rangle+\lambda h(p) \leq \lambda h(y), \quad \text { for all } y \in M .
$$

Proof. Let $y \in M$ and $p=\operatorname{prox}_{\lambda h}(x)$. Set $z_{\alpha}=\exp _{p} \alpha \exp _{p}^{-1} y$ for all $\alpha \in(0,1)$. Then by geodesic convexity of $h$ and (4.17), we get

$$
\lambda h(p) \leq \lambda h\left(z_{\alpha}\right)+\frac{1}{2} d^{2}\left(x, z_{\alpha}\right)-\frac{1}{2} d^{2}(x, p), \quad \text { for all } \alpha \in(0,1) .
$$

Consider the geodesic triangle $\triangle(p, y, x)$ and its comparison triangle $\triangle\left(p^{\prime}, y^{\prime}, x^{\prime}\right)$. Then by Lemma 2.1, we get

$$
d(p, y)=\left\|p^{\prime}-y^{\prime}\right\|, \quad d(y, x)=\left\|y^{\prime}-x^{\prime}\right\| \quad \text { and } \quad d(x, p)=\left\|x^{\prime}-p^{\prime}\right\| .
$$

Since $z_{\alpha}=\exp _{p} \alpha \exp _{p}^{-1} y$, the comparison point of $z_{\alpha}$ is $z_{\alpha}^{\prime}=\alpha y^{\prime}+(1-\alpha) p^{\prime}$. Let $\beta$ and $\beta^{\prime}$ denotes the angle at $p$ and $p^{\prime}$ respectively. Then by Lemma 2.2 (a), we have $\beta \leq \beta^{\prime}$, and hence $\cos \beta^{\prime} \leq \cos \beta$. By part (b) of Lemma 2.2, we get

$$
\begin{aligned}
d^{2}\left(x, z_{\alpha}\right) & \leq\left\|x^{\prime}-z_{\alpha}^{\prime}\right\|^{2}=\left\|\left(x^{\prime}-p^{\prime}\right)+\alpha\left(p^{\prime}-y^{\prime}\right)\right\|^{2} \\
& =\left\|x^{\prime}-p^{\prime}\right\|^{2}+\alpha^{2}\left\|p^{\prime}-y^{\prime}\right\|^{2}+2 \alpha\left\langle x^{\prime}-p^{\prime}, p^{\prime}-y^{\prime}\right\rangle_{\mathbb{R}^{2}} \\
& =\left\|x^{\prime}-p^{\prime}\right\|^{2}+\alpha^{2}\left\|p^{\prime}-y^{\prime}\right\|^{2}+2 \alpha\left\|x^{\prime}-p^{\prime}\right\|\left\|p^{\prime}-y^{\prime}\right\| \cos \beta^{\prime} \\
& \leq d^{2}(x, p)+\alpha^{2} d^{2}(p, y)+2 \alpha d(x, p) d(p, y) \cos \beta \\
& =d^{2}(x, p)+\alpha^{2} d^{2}(p, y)+2 \alpha\left\|\exp _{p}^{-1} x\right\|\left\|\exp _{y}^{-1} p\right\| \cos \beta \\
& =d^{2}(x, p)+\alpha^{2} d^{2}(p, y)+2 \alpha\left\|\exp _{p}^{-1} x\right\|\left\|P_{p, y} \exp _{y}^{-1} p\right\| \cos \beta \\
& =d^{2}(x, p)+\alpha^{2} d^{2}(p, y)+2 \alpha\left\langle\exp _{p}^{-1} x, P_{p, y} \exp _{y}^{-1} p\right\rangle \\
& =d^{2}(x, p)+\alpha^{2} d^{2}(p, y)-2 \alpha\left\langle\exp _{p}^{-1} x, \exp _{p}^{-1} y\right\rangle .
\end{aligned}
$$


From (4.18), we have

$$
\begin{aligned}
\lambda h(p) & \leq \lambda h\left(z_{\alpha}\right)+\frac{1}{2}\left\{d^{2}(x, p)+\alpha^{2} d^{2}(y, p)-2 \alpha\left\langle\exp _{p}^{-1} x, \exp _{p}^{-1} y\right\rangle\right\}-\frac{1}{2} d^{2}(x, p) \\
& =\lambda h\left(z_{\alpha}\right)-\alpha\left\langle\exp _{p}^{-1} x, \exp _{p}^{-1} y\right\rangle+\frac{\alpha^{2}}{2} d^{2}(y, p) \\
& \leq \lambda \alpha h(y)+\lambda(1-\alpha) h(p)-\alpha\left\langle\exp _{p}^{-1} x, \exp _{p}^{-1} y\right\rangle+\frac{\alpha^{2}}{2} d^{2}(y, p) .
\end{aligned}
$$

After simplification, we obtain

$$
\left\langle\exp _{p}^{-1} x, \exp _{p}^{-1} y\right\rangle+\lambda h(p) \leq \lambda h(y)-\frac{\alpha}{2} d^{2}(y, p) .
$$

Letting $\alpha \rightarrow 0$, we get $\left\langle\exp _{p}^{-1} x, \exp _{p}^{-1} y\right\rangle+\lambda h(p) \leq \lambda h(y)$.

Conversely, suppose that $\left\langle\exp _{p}^{-1} x, \exp _{p}^{-1} y\right\rangle+\lambda h(p) \leq \lambda h(y)$. Then

$$
\begin{aligned}
\lambda h(p)+\frac{1}{2} d^{2}(x, p) & \leq \lambda h(y)+\frac{1}{2} d^{2}(x, p)-\left\langle\exp _{p}^{-1} x, \exp _{p}^{-1} y\right\rangle+\frac{1}{2} d^{2}(p, y) \\
& =\lambda h(y)+\frac{1}{2} d^{2}(x, y) . \quad(\operatorname{by}(2.3))
\end{aligned}
$$

Therefore, $p=\operatorname{prox}_{\lambda h} x$.

By using the above lemma, we get the following result.

Lemma 4.6. For all $x \in M, y \in K$ and $\lambda>0$, the following inequality holds:

$$
\left\langle\exp _{\operatorname{prox}_{\lambda h}(x)}^{-1} x, \exp _{\operatorname{prox}_{\lambda h}(x)}^{-1} y\right\rangle \leq \lambda\left\{h(y)-h\left(\operatorname{prox}_{\lambda h}(x)\right)\right\} .
$$

Now, we are ready to propose two-step proximal point algorithm for solving equilibrium problem in the setting of Hadamard manifolds.

Let $\left\{\alpha_{n}\right\} \subset(0,+\infty)$ be a non-increasing sequence such that

$$
(A 1): \lim _{n \rightarrow \infty} \alpha_{n}=0 \text { and }(A 2): \sum_{n=0}^{\infty} \alpha_{n}=+\infty .
$$

Algorithm 4.6. Choose arbitrary $x_{0}, y_{0} \in K$ and a non-increasing sequence $\left\{\alpha_{n}\right\} \subset(0,+\infty)$ such that the conditions (A1) and (A2) hold. Assume that $x_{n}, y_{n} \in K$ are known, calculate $x_{n+1}$ and $y_{n+1}$ as follows:

Step 1. Compute

$$
x_{n+1}=\operatorname{prox}_{\alpha_{n} f\left(y_{n}, \cdot\right)}\left(x_{n}\right) .
$$

If $x_{n+1}=y_{n}=x_{n}$, then stop and $x_{n}$ is the solution of problem EP (2.6). Otherwise,

Step 2. Compute

$$
y_{n+1}=\operatorname{prox}_{\alpha_{n+1} f\left(y_{n}, \cdot\right)}\left(x_{n+1}\right) .
$$

Set $n=: n+1$ and go back Step 1 .

Definition 4.6. A bifunction $f: K \times K \rightarrow \mathbb{R}$ is said to satisfies Lipschitz-type condition on $K$ if there exist $\lambda_{1}, \lambda_{2}>0$ such that

$$
f(x, y)+f(y, z) \geq f(x, z)-\lambda_{1} d^{2}(x, y)-\lambda_{2} d^{2}(y, z), \quad \text { for all } x, y, z \in K .
$$

This kind of the condition was first used in [20] to study auxiliary principle for EP in the setting of reflexive Banach spaces, and later it was used by Hieu [11].

Now we study the convergence of the sequences generated by Algorithm 4.6.

Theorem 4.7. Assume that the bifunction $f: K \times K \rightarrow \mathbb{R}$ satisfies the following conditions.

(i) $f(x, x)=0$ for all $x \in K$; 
(ii) $f$ is strongly pseudomonotone on $K$;

(iii) $f$ satisfies the Lipschitz-type condition on $K$;

(iv) $f(x, \cdot)$ is geodesic convex and lower semicontinuous and $f(\cdot, y)$ is geodesic hemicontinuous on $K$.

If conditions (A1) and (A2) hold, then the sequences $x_{n}$ and $y_{n}$ generated by Algorithm 4.6 converges to a unique solution $\bar{x}$ of EP (2.6).

Proof. Consider the points $x_{n}, x_{n+1}$ and $\bar{x}$, and using (2.3), we obtain

$$
d^{2}\left(x_{n}, x_{n+1}\right)+d^{2}\left(x_{n+1}, \bar{x}\right)-2\left\langle\exp _{x_{n+1}}^{-1} x_{n}, \exp _{x_{n+1}}^{-1} \bar{x}\right\rangle \leq d^{2}\left(x_{n}, \bar{x}\right) .
$$

Again by considering the points $x_{n}, y_{n}$ and $x_{n+1}$, and using (2.3), we get

$$
d^{2}\left(x_{n}, y_{n}\right)+d^{2}\left(y_{n}, x_{n+1}\right)-2\left\langle\exp _{y_{n}}^{-1} x_{n}, \exp _{y_{n}}^{-1} x_{n+1}\right\rangle \leq d^{2}\left(x_{n}, x_{n+1}\right) .
$$

By combining (4.24) and (4.25), we have

$$
\begin{aligned}
d^{2}\left(x_{n+1}, \bar{x}\right) \leq & d^{2}\left(x_{n}, \bar{x}\right)-d^{2}\left(x_{n}, x_{n+1}\right)+2\left\langle\exp _{x_{n+1}}^{-1} x_{n}, \exp _{x_{n+1}}^{-1} \bar{x}\right\rangle \\
\leq & d^{2}\left(x_{n}, \bar{x}\right)-\left\{d^{2}\left(x_{n}, y_{n}\right)+d^{2}\left(y_{n}, x_{n+1}\right)-2\left\langle\exp _{y_{n}}^{-1} x_{n}, \exp _{y_{n}}^{-1} x_{n+1}\right\rangle\right\} \\
& +2\left\langle\exp _{x_{n+1}}^{-1} x_{n}, \exp _{x_{n+1}}^{-1} \bar{x}\right\rangle \\
\leq & d^{2}\left(x_{n}, \bar{x}\right)-d^{2}\left(x_{n}, y_{n}\right)-d^{2}\left(y_{n}, x_{n+1}\right)+2\left\langle\exp _{y_{n}}^{-1} x_{n}, \exp _{y_{n}}^{-1} x_{n+1}\right\rangle \\
& +2\left\langle\exp _{x_{n+1}}^{-1} x_{n}, \exp _{x_{n+1}}^{-1} \bar{x}\right\rangle .
\end{aligned}
$$

From (4.22) and Lemma 4.6, we obtain

$$
\alpha_{n}\left\{f\left(y_{n}, y\right)-f\left(y_{n}, x_{n+1}\right)\right\} \geq\left\langle\exp _{x_{n+1}}^{-1} x_{n}, \exp _{x_{n+1}}^{-1} y\right\rangle, \quad \text { for all } y \in K \text {. }
$$

Setting $y=\bar{x} \in K$, we get

$$
\alpha_{n}\left\{f\left(y_{n}, \bar{x}\right)-f\left(y_{n}, x_{n+1}\right)\right\} \geq\left\langle\exp _{x_{n+1}}^{-1} x_{n}, \exp _{x_{n+1}}^{-1} \bar{x}\right\rangle .
$$

Now, from (4.23) and Lemma 4.6, we have

$$
\alpha_{n}\left\{f\left(y_{n-1}, y\right)-f\left(y_{n-1}, y_{n}\right)\right\} \geq\left\langle\exp _{y_{n}}^{-1} x_{n}, \exp _{y_{n}}^{-1} y\right\rangle, \quad \text { for all } y \in K \text {. }
$$

Setting $y=x_{n+1} \in K$, we get

$$
\alpha_{n}\left\{f\left(y_{n-1}, x_{n+1}\right)-f\left(y_{n-1}, y_{n}\right)\right\} \geq\left\langle\exp _{y_{n}}^{-1} x_{n}, \exp _{y_{n}}^{-1} x_{n+1}\right\rangle .
$$

On employing (4.27) and (4.28), inequality (4.26) yields

$$
\begin{aligned}
d^{2}\left(x_{n+1}, \bar{x}\right) \leq & d^{2}\left(x_{n}, \bar{x}\right)-d^{2}\left(x_{n}, y_{n}\right)-d^{2}\left(y_{n}, x_{n+1}\right) \\
& +2 \alpha_{n}\left\{f\left(y_{n}, \bar{x}\right)-f\left(y_{n}, x_{n+1}\right)\right\}+2 \alpha_{n}\left\{f\left(y_{n-1}, x_{n+1}\right)-f\left(y_{n-1}, y_{n}\right)\right\} \\
\leq & d^{2}\left(x_{n}, \bar{x}\right)-d^{2}\left(x_{n}, y_{n}\right)-d^{2}\left(y_{n}, x_{n+1}\right) \\
& +2 \alpha_{n}\left\{f\left(y_{n}, \bar{x}\right)-f\left(y_{n}, x_{n+1}\right)+f\left(y_{n-1}, x_{n+1}\right)-f\left(y_{n-1}, y_{n}\right)\right\} .
\end{aligned}
$$

Since $f$ satisfies Lipschitz-type condition, there exist $c_{1}>0$ and $c_{2}>0$ such that

$$
f\left(y_{n-1}, y_{n}\right)+f\left(y_{n}, x_{n+1}\right) \geq f\left(y_{n-1}, x_{n+1}\right)-c_{1} d^{2}\left(y_{n-1}, y_{n}\right)-c_{2} d^{2}\left(y_{n}, x_{n+1}\right) .
$$

By using this inequality and (4.29), we obtain

$$
\begin{aligned}
d^{2}\left(x_{n+1}, \bar{x}\right) \leq d^{2}\left(x_{n}, \bar{x}\right)-d^{2}\left(x_{n}, y_{n}\right)- & d^{2}\left(y_{n}, x_{n+1}\right)+2 \alpha_{n} f\left(y_{n}, \bar{x}\right) \\
& +2 \alpha_{n} c_{1} d^{2}\left(y_{n-1}, y_{n}\right)+2 \alpha_{n} c_{2} d^{2}\left(y_{n}, x_{n+1}\right)
\end{aligned}
$$

Since $\bar{x}$ is a solution of problem EP (2.6), so $f\left(\bar{x}, y_{n}\right) \geq 0$. By using the strong pseudomonotonicity properties of $f$, we have

$$
f\left(y_{n}, \bar{x}\right) \leq-\lambda d^{2}\left(y_{n}, \bar{x}\right), \quad \text { for some } \lambda>0 .
$$


Note that

$$
d^{2}\left(y_{n-1}, y_{n}\right) \leq 2 d^{2}\left(y_{n-1}, x_{n}\right)+2 d^{2}\left(x_{n}, y_{n}\right)
$$

By combining (4.30), (4.31) and (4.32), we obtain

$$
\begin{aligned}
d^{2}\left(x_{n+1}, \bar{x}\right) \leq & d^{2}\left(x_{n}, \bar{x}\right)-d^{2}\left(x_{n}, y_{n}\right)-d^{2}\left(y_{n}, x_{n+1}\right)-2 \alpha_{n} \lambda d^{2}\left(y_{n}, \bar{x}\right)+4 \alpha_{n} c_{1} d^{2}\left(y_{n-1}, x_{n}\right) \\
& +4 \alpha_{n} c_{1} d^{2}\left(y_{n}, x_{n}\right)+2 \alpha_{n} c_{2} d^{2}\left(y_{n}, x_{n+1}\right) \\
= & d^{2}\left(x_{n}, \bar{x}\right)-\left(1-4 \alpha_{n} c_{1}\right) d^{2}\left(x_{n}, y_{n}\right)-\left(1-2 \alpha_{n} c_{2}\right) d^{2}\left(y_{n}, x_{n+1}\right)-2 \alpha_{n} \lambda d^{2}\left(y_{n}, \bar{x}\right) \\
& +4 \alpha_{n} c_{1} d^{2}\left(y_{n-1}, x_{n}\right) .
\end{aligned}
$$

Adding the term $4 \alpha_{n+1} c_{1} d^{2}\left(y_{n}, x_{n+1}\right)$ to both sides of (4.33), we get

$$
\begin{aligned}
d^{2} & \left(x_{n+1}, \bar{x}\right)+4 \alpha_{n+1} c_{1} d^{2}\left(y_{n}, x_{n+1}\right) \\
\leq & d^{2}\left(x_{n}, \bar{x}\right)+4 \alpha_{n+1} c_{1} d^{2}\left(y_{n}, x_{n+1}\right)-\left(1-4 \alpha_{n} c_{1}\right) d^{2}\left(x_{n}, y_{n}\right) \\
& -\left(1-2 \alpha_{n} c_{2}\right) d^{2}\left(y_{n}, x_{n+1}\right)-2 \alpha_{n} \lambda d^{2}\left(y_{n}, \bar{x}\right)+4 \alpha_{n} c_{1} d^{2}\left(y_{n-1}, x_{n}\right) \\
= & d^{2}\left(x_{n}, \bar{x}\right)+4 \alpha_{n} c_{1} d^{2}\left(y_{n-1}, x_{n}\right)-\left(1-4 \alpha_{n} c_{1}\right) d^{2}\left(x_{n}, y_{n}\right) \\
& -\left(1-2 \alpha_{n} c_{2}-4 \alpha_{n+1} c_{1}\right) d^{2}\left(y_{n}, x_{n+1}\right)-2 \alpha_{n} \lambda d^{2}\left(y_{n}, \bar{x}\right),
\end{aligned}
$$

which yields that

$$
a_{n+1} \leq a_{n}-b_{n}-\alpha_{n} c_{n}
$$

where $a_{n}=d^{2}\left(x_{n}, \bar{x}\right)+4 \alpha_{n} c_{1} d^{2}\left(y_{n-1}, x_{n}\right), b_{n}=\left(1-4 \alpha_{n} c_{1}\right) d^{2}\left(x_{n}, y_{n}\right)+\left(1-2 \alpha_{n} c_{2}-\right.$ $\left.4 \alpha_{n+1} c_{1}\right) d^{2}\left(y_{n}, x_{n+1}\right)$ and $c_{n}=2 \lambda d^{2}\left(y_{n}, \bar{x}\right)$. Therefore, $a_{n} \geq 0$ and $c_{n} \geq 0$ for all $n \geq 0$.

Now we claim that $b_{n} \geq 0$ for all $n \geq 0$. Let $\delta \in(0,1)$ be a fixed number. Since $\alpha_{n} \rightarrow 0$, there exists $n_{0} \geq 0$ such that for all $n \geq n_{0}$, we have

$$
0<\delta \leq 1-4 \alpha_{n} c_{1}<1 \text { and } 0<\delta \leq 1-2 \alpha_{n} c_{2}-4 \alpha_{n+1} c_{1}<1 .
$$

Therefore, $b_{n} \geq 0$ for all $n \geq n_{0}$. Thus from (4.35), we get $0 \leq a_{n+1} \leq a_{n}$ for all $n \geq$ $n_{0}$, which implies that $\lim _{n \rightarrow \infty} a_{n}$ exists in $\mathbb{R}$. It follows from the definition of $a_{n}$ that the sequence $\left\{d^{2}\left(x_{n}, \bar{x}\right)\right\}$ is bounded, and hence $\left\{x_{n}\right\}$ is also bounded. The relation (4.35) can be rewritten as

$$
b_{n}+\alpha_{n} c_{n} \leq a_{n}-a_{n+1}, \quad \text { for all } n \geq n_{0} .
$$

Let $N \geq n_{0}$ be fixed. Taking $n=n_{0}, \ldots, N$ in inequality (4.37) and summing up the resultant inequalities, we get

$$
\sum_{n=n_{0}}^{N} b_{n}+\sum_{n=n_{0}}^{N} \alpha_{n} c_{n} \leq a_{n_{0}}-a_{N+1} \leq a_{n_{0}} .
$$

Now, letting $N \rightarrow \infty$, we obtain

$$
\sum_{n=n_{0}}^{\infty} b_{n}+\sum_{n=n_{0}}^{\infty} \alpha_{n} c_{n}<+\infty
$$

which implies that

$$
(H 1): \quad \sum_{n=n_{0}}^{\infty} b_{n}<+\infty \text { and }(H 2): \quad \sum_{n=n_{0}}^{\infty} \alpha_{n} c_{n}<+\infty .
$$

Thus from the definition of $b_{n}$, relation (4.36) and (H1), we obtain

$$
\lim _{n \rightarrow \infty} d^{2}\left(x_{n}, y_{n}\right)=\lim _{n \rightarrow \infty} d^{2}\left(y_{n}, x_{n+1}\right)=0 .
$$


Since $\left\{x_{n}\right\}$ is bounded, the inequality (4.38) implies that $\left\{y_{n}\right\}$ is bounded. As $\alpha_{n} \rightarrow 0$ and $\lim _{n \rightarrow \infty} a_{n} \in \mathbb{R}$, from the definition of $a_{n}$, we get

$$
\lim _{n \rightarrow \infty} d^{2}\left(x_{n}, \bar{x}\right) \in \mathbb{R} .
$$

Using (H2) and $\sum_{n=n_{0}}^{\infty} \alpha_{n}=+\infty$, we get $\liminf _{n \rightarrow \infty} c_{n}=0$. Therefore from the definition of $c_{n}$ and $\lambda>0$, we have $\liminf _{n \rightarrow \infty} d^{2}\left(y_{n}, \bar{x}\right)=0$. This together with relation (4.38) gives $\liminf _{n \rightarrow \infty} d^{2}\left(x_{n}, \bar{x}\right)=0$. Hence from (4.39), we have $\lim _{n \rightarrow \infty} d^{2}\left(x_{n}, \bar{x}\right)=0$, that is, $x_{n} \rightarrow \bar{x}$ as $n \rightarrow \infty$. By using relation (4.38), we obtain that the sequence $\left\{y_{n}\right\}$ is also convergent.

Remark 4.7. (a) Algorithm 4.6 is the extension of Algorithm 3.1 in [11] from Hilbert spaces to Hadamard manifolds.

(b) Algorithm 4.6 generalizes Algorithm 1 in [7] in the following ways:

(i) In the convergence result of Algorithm 1 in [7], the sequence of step-size is bounded and depends on the modulus of the Lipschitz-type constant function, which can make restrictions in applications because Lipschitz-type constants are often unknown or difficult to approximate, whereas in our convergence result the sequence of step-size is independent of the modulus of the Lipschitz-type constants as well as the modulus of the strong pseudomontonicity constant of the equilibrium bifunction, that is, the construction of solution approximations and the proof of its convergence can be done without the prior knowledge of the modulus of Lipschitztype constants and strong pseudomonotonicity constant of the equilibrium bifunction.

(ii) A little difference in comparison with Algorithm 1 in [7] is that in Algorithm 4.6 we used two distinct step-sizes while only one value of equilibrium bifunction at current approximation needs to proceed per each iteration.

Acknowledgment. The first author is grateful to King Fahd University of Petroleum and Minerals, Dhahran, Saudi Arabia for providing excellent research facilities. In this research, second author was supported by a research grant DST-SERB No. EMR/2016/005124.

\section{REFERENCES}

[1] Ansari, Q. H.; Islam, M.; Yao, J. C. Nonsmooth convexity and monotonicity in terms of a bifunction on Riemannian manifolds. J. Nonlinear Convex Anal. 18 (2017), no. 4, 743-762.

[2] Ansari, Q. H.; Islam, M.; Yao, J.-C. Nonsmooth Variational Inequalities on Hadamard Manifolds. Appl. Anal. 99 (2020), no. 2, 340-358 and 359-360-Correction

[3] Bauschke, H. H.; Combettes, P. L. Convex Analysis and Monotone Operator Theory in Hilbert Spaces. CMS Books in Mathematics/Ouvrages de Mathématiques de la SMC. Springer, New York, 2011.

[4] Bento, G. C.; Cruz Neto, J. X. Finite termination of the proximal point method for convex functions on Hadamard manifolds. Optimization, 63 (2014), no. 9, 1281-1288.

[5] Blum, E.; Oettli, W. From optimization and variational inequalities to equilibrium problems. Math. Stud. 63 (1994), 123-145.

[6] Colao, V.; López, G.; Marino, G.; Martín-Márquez, V. Equilibrium problems in Hadamard manifolds. J. Math. Anal. Appl. 388 (2012), 61-77.

[7] Cruz Neto, J. X.; Santos, P. S. M.; Soares Jr, P. A. An extragradient method for solving equilibrium problems on Hadamard manifolds. Optim Lett. 10 (2016), 1327-1336.

[8] do Carmo, M. P. Riemannian Geometry Birkhauser, Boston, 1992.

[9] Ferreira, O. P., Oliveira, P. R. Proximal point algorithm on Riemannian manifolds. Optimization 51 (2002), 257-270.

[10] Flores-Bazán, F. Existence theory for finite-dimensional pseudomonotone equilibrium problems. Acta Appl. Math. 77 (2003), 249-297.

[11] Hieu, D. V. Convergence analysis of a new algorithm for strongly pseudomonotone equilibrium problems. Numer. Algor. 77 (2018), 983-1001. 
[12] Hieu, D. V.; Cho, Y. C.; Xiao, Y.-b. Modified extragradient algorithms for solving equilibrium problems. Optimization 67 (2018), 2003-2029.

[13] Hosseini, S.; Pouryayevali, M. R. Generalized gradients and characterizations of epi-Lipschitz sets in Riemannian manifolds, Nonlinear Anal. 74 (2011), 3884-3895.

[14] Kassay, G. The Equilibrium Problem and Related Topics. Cluj-Napoca Risoprint, Cluj-Napoca, 2000.

[15] Kim, J. K.; Anh, P. N.; Hyun, H. G. A proximal point-type algorithm for pseudomonotone equilibrium problems. Bull. Korean Math. Soc. 49 (2012), no. 4, 749-759.

[16] Knaster, B.: Kuratowski, C.: Mazurkiewicz, S. Einbeweis des fixpunktsatzes für n-dimensionale simplexes, Fund. Math. 14 (1929), 132-137.

[17] Li, C.; López, G.; Martín-Márquez, V. Iterative algorithms for nonexpansive mappings on Hadamard manifolds, Taiwanese Journal of Mathematics, 14 (2010) no. 2, 541-559.

[18] Li, C.; Yao, J. C. Variational inequalities for set-valued vector fields on Riemannian manifolds: convexity of the solution set and the proximal point algorithm, SIAM J. Control Optim. 50 (2012), no. 4, 2486-2514.

[19] Lyashko, S.; Semenov, V. V. A new two-step proximal algorithm of solving the problem of equilibrium programming, In: Optimization and Its Applications in Control and Data Sciences, B. Goldengorin (editor), Springer International Publishing Switzerland, (2016), 315-325.

[20] Mastroeni, G. On auxiliary principle for equilibrium problems. Publicatione del Dipartmento di Mathematica dell, Universita di Pis, 3 (2000), 1244-1258.

[21] Németh, S. Z. Variational inequalities on Hadamard manifolds. Nonlinear Anal., 52 (2003), 1491-1498.

[22] Reich, S. Strong convergence theorems for resolvent accretive operators in Banach spaces. J. Math. Anal. Appl. 75 (1980), 287-292.

[23] Sakai, T. Riemannian Geometry. American Mathematical Society, Providence, RI, 1996.

[24] Spivak, M. Calculus on Manifolds: A Modern Approach to Classical Theorems of Advanced Calculus. W. A. Benjamin, Inc, New York, Amsterdam, 1965.

[25] Tang, Guo-ji; Zhou, Li-wen; Huang Nan-jing. Existence results for a class of hemivariational inequality problems on Hadamard manifolds. Optimization 65 (2016), no. 7, 1451-1461.

[26] Udriste, C. Convex Functions and Optimization Methods on Riemannian Manifolds. Kluwer Academic, Dordrecht, 1994.

[27] Zhou, L.-W.; Xiao. Y.-B.; Huang, N.-J. New Characterization of geodesic convexity on Hadamard manifolds with applications. J. Optim. Theory Appl. 172 (2016), no. 3, 824-844.

${ }^{1}$ Department of Mathematics and Statistics

King Fahd University of Petroleum and Minerals

DHAHRAN, SAUDi ARABIA

Email address: homidan@kfupm.edu.sa

${ }^{2}$ Department of Mathematics

ALigarh Muslim University

ALIGARH, INDIA

Email address: qhansariegmail.com

Email address: monirul.amuegmail.com 